\title{
Effect of A Navicular Support Foot Orthosis on Resting Calcaneal Standing Position Angle and Navicular Height in Flat Foot Children
}

\author{
Seong Hee Kim ${ }^{1}$, Jang Hwan $\mathrm{Kim}^{2}$, and Kang Seong Lee ${ }^{3 *}$ \\ Dept. of Rehabilitation Science Graduate School, Hanseo University, Korea \\ ${ }^{1} 0167073758 @ n a v e r . c o m,{ }^{2}$ profpo@hanseo.ac.kr, ${ }^{3}$ cpo114@hanseo.ac.kr
}

\begin{abstract}
This study aims to compare resting calcaneal standing position and navicular height, which are used as diagnostic factors for pes planus, before and after the use of an orthotic with a navicular support in children who had severe flexible pes planus accompanied by navicular drop. Participants wore the navicular orthotic support for more than 8 hours a day. Measurements were taken before putting on the foot apparatus and at 6 and 12 months after; all measurements were taken while the children were not wearing the orthosis. After using the orthosis with added navicular support, the resting calcaneal standing position increased and the navicular height also increased. The increase in the navicular height suggested that the navicular bone collapsed due to the severe flexible pes planus. Our research findings showed that the use of orthosis with navicular support had a good effect for treating children with severe flexible pes planus accompanied by navicular drop. By comparing the long-term effects of orthotics used in this study with existing ones currently used for treatment of flexible pes planus, we can now determine the effects of orthotics with added navicular support and we can have a positive effect on the treatment of navicular drop when accompanied by the symptoms of pes planus. This study showed that orthotics with navicular support increased the resting calcaneal standing position and navicular height in children with severe flexible pes planus.
\end{abstract}

Keywords: Resting calcaneal standing position, Navicular support, Pes planus, Orthotic

\section{Introduction}

The foot is divided into three parts: the rearfoot, midfoot, and forefoot. The rearfoot is composed of the talus and calcaneus; the midfoot is composed of the navicular, cuboid, and cuneiforms; and the forefoot is composed of metatarsal bones and phalanges [1]. The longitudinal and transverse arches on the bottom of the foot provide the most stable weight distribution, biomechanically assisting in protection of the body and reducing fatigue when either standing or walking for a long time [2]. As it grows after birth, the medial longitudinal arch of the foot appears to be reduced in terms of both subcutaneous fatty tissue and flexibility of the ligaments, beginning at around 6 years of age and completing its development at around 12 years of age [3]. A flat foot is caused by structural loss of the medial longitudinal arch, and it is a common result of various factors regardless of patient age and the exact etiology [4]. Structural loss of the medial arch results in flatfoot, which is very common due to various causes regardless of age and disease of the foot. Furthermore, the navicular bone needs to be corrected because it is situated on the apex of the medial longitudinal arch, and the curvature

Article history:

Received (August 9, 2019), Review Result (September 27, 2019), Accepted (November 6, 2019) 
of the longitudinal arch is determined by the angle between the long axis of the metatarsal bone and the height from the ground of the calcaneus [5].

Children with flat feet do not correct themselves as they grow up, but suffer from pain as adults, and pronation should be treated as soon as possible with the most common modifications in children who often weight-bear [6][7]. Foot ligaments, such as the plantar calcaneonavicular ligament, plantar fascia, and deltoid ligament, maintain the stability of the medial arch, lower the bones and cuboid, and shift the skeletal structure away from the midline, and other neuromuscular disease such as cerebral palsy. In order to alleviate the symptoms in the flat children, various foot assisters have been applied to study the effects. Most of the studies were on the medial arch support rather than on the Blake internal foot assist. As part of a research project to develop foot orthoses to relieve symptoms, Sinha et al. [8] measured pain in the forefoot, midfoot, and hindfoot of 81 children with bilateral pes planus after wearing a medial longitudinal arch support, and Robert et al. [9] examined 22 subjects (6 to 57 years old) with pes planus who were wearing custom foot orthoses. They noted the changes in the lateral talocalcaneal angle during their X-ray imaging assessment of the effect of a medial longitudinal arch support on flexible pes planus. The study's results were consistent with Perry's [10] results, revealing that the talo-metatarsal angle was significantly decreased in the flat foot as compared to the normal foot through the use of a sagittal view radiograph, an approach that has been recognized as the standard when making a diagnosis of flat foot. Kanatli et al. [11] also reported that physical and radiographic examinations are used to assess flatfeet in children with radiographs used for direct observation of the bones and arch of the foot. The Blake inversion foot orthosis, which was used to treat flatfeet in children, has a structure that supports the medial of the arch.

Blake inversion foot orthoses are characterized by gypsum bonding in the unweighted state and modify the posterior forefoot and forefoot eversion, bringing the foot arch closer to normal. In Blake's study of a foot orthosis used for the treatment of pes planus, there are insufficient data reported on the effect of the orthosis in regards to correction of the navicular bone. Therefore, the objective of this study was to evaluate the difference in height for both the resting calcaneal standing position angle and the navicular bone in children with flat foot after wearing a Blake inversion foot orthosis with additional navicular support.

\section{Methods}

\subsection{Subjects}

Ten subjects who were diagnosed with severe bilateral flexible pes planus and were observed to have a descending navicular bone were selected for this study from the podiatry clinic of the Chungnam National University hospital. The inclusion criteria were as follows: children with severe flexible pes planus and a descending navicular bone, no prior prescription of a foot orthosis with a navicular support, no other orthopedic conditions or abnormalities, and able to wear a foot orthosis for more than 8 hours a day. Exclusion criteria were rheumatism; fractures; spinal tumors; a history of other orthopedic surgeries; chronic pain disorder; neuromuscular compression; the presence of any major circulatory, respiratory, neurological, or heart disorders; cognitive deficits; infections; and prior treatment at a different institution. All subjects were informed of the study's objectives and procedures prior to the beginning of the experiment, and they provided written informed consent. The characteristics of the participants include in the study are shown in [Table 1]. 
Table 1. General characteristics of the participants

\begin{tabular}{|c|c|}
\hline & Average \pm standard deviation \\
\hline Sex & Male 6, Female 4 \\
\hline Age, years & $10.8 \pm 1.61$ \\
\hline Height, $\mathrm{m}$ & $148.6 \pm 2.61$ \\
\hline Weight, kg & $36.9 \pm 2.23$ \\
\hline
\end{tabular}

\subsection{Methods}

\subsubsection{The experimental procedure}

The participants were prescribed navicular support-type foot orthoses [Figure 2], and their measurements were compared before and after regular use of the foot orthosis. The foot orthosis was fabricated by a skilled orthotist after making a negative plaster model. In order to support the lowered navicular bone, the phalange of the existing Blake inner foot brace was extended to the cuboid area and the pad was minimized by the pressure of the phalange. The application of foot orthoses on the phalanges will also provide the effect of widening the support surface to distribute the pressure. In order to accurately support the navicular area through the phalange, the navicular area was marked in the bonding process so that it appeared in the benign model, and the height of the phalange was adjusted. The width was $1 / 3$ of the total length of the inner shell. Cork cover was applied to the scapular support foot brace to make it stable, and the cover length was extended to the forefoot to allow the foot brace to be seated in the shoe.

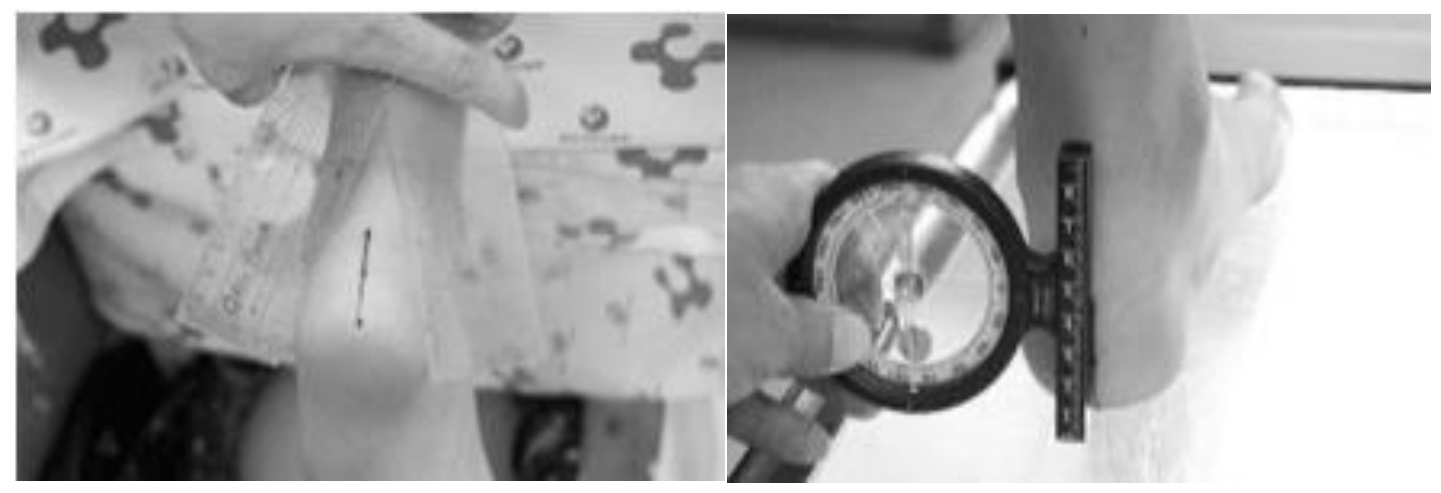

Figure 1. Resting calcaneal standing position test

A more effective foot assist device was designed. Blake inversion foot orthoses are characterized by gypsum bonding under unweighted support and modifying model to create forefoot inversion. All participants wore the navicular support-type foot orthosis for more than 8 hours per day during the study. X-ray images in the lateral view were taken in the standing position to allow for accurate measurement of the resting calcaneal standing angle and the medial longitudinal arch, allowing for measurement of the inclination of the calcaneus before wearing the foot orthosis. The height of the navicular bone was measured on X-ray images taken before the use of the foot orthosis and then at 6 and 12 months after regularly wearing 
the foot orthosis. The actual measurements were taken while the child was not wearing the foot orthosis.

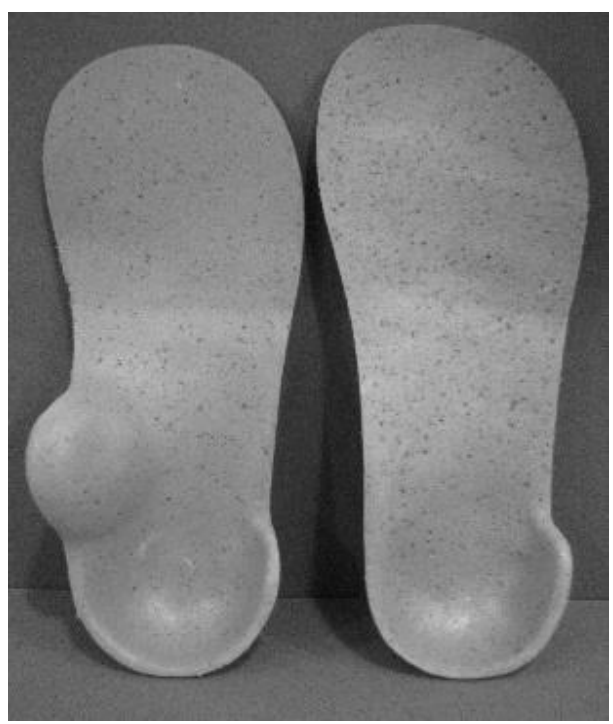

Figure 2. Navicular support foot orthosis

\subsubsection{Measurement equipment and variables}

Lateral X-rays were performed in the standing position to accurately measure the standing calcaneus angle and the arch, which measured the inclination of the calcaneus before wearing the Blake inner foot brace. X-ray images were used to measure the resting calcaneal standing position angle, and the height of the navicular bone. All measurements were taken without wearing foot braces. Descriptions of each measurement are as follows:

(1) Measurement of height and weight

An automatic electric scale (BSM 330, Biospace, Seoul, Korea) was used for measuring the participants' height and weight.

(2) Resting calcaneal standing position

The participant was placed in the prone position without loading, and we drew a line that bisected the calcaneus of the heel. Then, the angle between the line and the ground was measured while the participant was standing upright with loading weight [Figure 1].

(3) Navicular height

The height was measured vertically from the center of the navicular bone to the ground in the lateral view X-ray image.

\subsubsection{Data analysis}

All data were analyzed using SPSS 18.0 for Windows. The Kolmogorov-Smirnov (K-S) test validated the assumption of a normal distribution based on the general characteristics of the participants. The resting calcaneal standing angle, talocalcaneal angle, talometatarsal angle, and height of the navicular bone were recorded, and the data of the resting calcaneal standing angle and X-ray measurements were compared before and 6 and 12 months after the regular use of the foot orthosis. To verify the differences among these measurements, repeated measures analysis of variance was performed. Bonferroni's post hoc test was also performed if there was a statistical difference (with $\alpha=.05 / 3=.017$ ). All statistical significance levels were set to $\alpha=.05$. 


\section{Results}

The changes in resting calcaneal standing angle and the height of the navicular bone 12 months after the participants started using the navicular support foot orthosis are shown in [Table 2]. The post hoc analysis showed that the resting calcaneal standing angle was significantly decreased from $-8.70^{\circ}$ to $-6.30^{\circ}$ at 6 months and $-4.85^{\circ}$ at 12 months $(\mathrm{p}<.001)$. Furthermore, the height of the navicular bone was significantly increased from $2.185 \mathrm{~cm}$ to $2.380 \mathrm{~cm}$ after 6 months and to $2.585 \mathrm{~cm}$ after 12 months $(\mathrm{p}<.001)$.

Table 2. Mean differences among the variables over time

\begin{tabular}{|c|c|c|c|c|}
\hline & Prior & $\begin{array}{c}\text { After 6 } \\
\text { months }\end{array}$ & $\begin{array}{c}\text { After 12 } \\
\text { months }\end{array}$ & $\mathrm{p}$ \\
\hline $\begin{array}{c}\text { The difference in the } \\
\text { resting calcaneal } \\
\text { standing angle }\end{array}$ & $-8.7 \pm 1.45$ & $-6.3 \pm 1.02$ & $-4.85 \pm 0.570$ & $<.001^{*+}$ \\
\hline $\begin{array}{c}\text { The height of the } \\
\text { navicular bone }\end{array}$ & $2.185 \pm 0.54$ & $2.38 \pm 0.68$ & $2.585 \pm 0.970$ & $<.001^{*+}$ \\
\hline
\end{tabular}

\section{Discussion}

The purpose of this study was to evaluate the effect of a foot orthosis for the treatment of pes planus in children by comparing their measurements before and after regular use of a Blake inversion foot orthosis with navicular support. Both the resting calcaneal standing angle and the height of the navicular bone were significantly increased when the navicular support foot orthosis was worn.

The results of wearing the navicular support foot orthosis on 20 feet of 10 children with flexible pes planus showed the resting calcaneal standing angle was decreased by $2.40^{\circ}$ after 6 months and $3.85^{\circ}$ after 12 months. In addition, the height of the navicular bone increased by $0.2 \mathrm{~cm}$ after 6 and 12 months of regular use of the orthosis.

There are no statements from accurate or convincing studies on how severe pes planus should be before it is treated and there are differences in criteria across studies, but Root [12] found that normal inverted resting calcaneal standing angles are in the range of $-4^{\circ}$ to around $-6^{\circ}$. On the other hand, LeLievere [13] stated that treatment should be started if there is a deviation of $-5^{\circ}$ in adults. A previous study in children with cerebral palsy using X-ray images showed a $0.45 \mathrm{~cm}$ increased height of the navicular bone after wearing medial longitudinal arch supports over 3 months [14]. Kulcu et al. [15] reported that flatfeet had no effect on the gait of the patient before compared to after the use of the foot brace or on the radiological results. A study of the effect of decreasing the height of the navicular bone on the clinical evaluation of lower limb alignment found that lowering the height of the navicular bone was correlated with the quadriceps angle and hip internal rotation angle [16], and $5^{\circ}$ of Cobb's angle and $2^{\circ}$ of the resting calcaneal standing angle improved in 38 children with scoliosis after the use of a Blake inversion foot orthosis [17]; these findings are consistent with our results. The navicular bone, supporting the structure of the medial arch, is descending and protruding inward in cases of severe pes planus [18]. Using X-ray imaging, Roth et al. [19] measured the talonavicular angle of a child with a flexible flat foot who had undergone surgery. The surgery may reduce the 
angle, but to a limit that still does not fall within the normal range. This treatment is unlikely to be different from the results of continuously wearing the foot brace with navicular support, as in this study. In the current study, an increase in the resting calcaneal standing angle through the use of a foot orthosis with hindfoot inversion and forefoot eversion plus navicular support improved the symptoms of flat foot due to less collapse of the calcaneus medially and an increase in the navicular height. In addition, the foot orthosis structure supports the medical longitudinal arch so that the inwardly protruding navicular bone could have an effect on children with pes planus by correcting the resting calcaneal standing angle.

The limitations of this study are that the effectiveness of different types of foot orthoses could not be compared and the sample size was relatively small. The effect of this navicular support-type foot orthosis could be more reliably investigated through a long-term comparison of it with the original Blake inversion foot orthosis. While applying the navicular foot brace, some modifications were needed for the appearance and contact surface of the external brace. If we compare the effect of wearing the existing Blake inversion foot orthosis with the navicular support designed in this study over the long-term, the effect of the navicular foot brace can be investigated more reliably. For further research, a long-term comparative study is needed to test the navicular foot support with the comfortable contact surface and the existing foot support in a larger number of subjects with flat feet.

\section{Conclusion}

This study evaluated the effects of a Blake inversion foot orthosis for the treatment of children's pes planus by comparing measurements before its use with after its use for 6 and 12 months. The resting calcaneal standing angle was significantly decreased after regular use of this orthosis. This navicular support-type foot orthosis can be considered as a new treatment option for pes planus in children.

\section{References}

[1] D.A. Neumann, "Kinesiology of the musculaoskeletal system: Foundation for physical rehabilitation," Philadelpia: Mosby, (2002)

[2] D. J. Magee, “Orthopedic Physical Assessment,” WB, Saunders, Philadelphia, (2008)

[3] A. Garcı-Rodrıguez, F. Martın-Jiménez, M. Carnero-Varo, E. Gómez-Gracia, J. Gómez-Aracena, and J. Fernández-Crehuet, "Flexible flat feet in children:a red problem?” Pediatrics, vol.103, pp.84-87, (1999)

[4] M. Pfeiffer, R. Kotz, T. Ledl, G. Hauser, and M. Sluga, "Prevalence of flat foot in preschool aged children," Pediatrics, vol.118, no.2, pp.634-639, (2006) DOI: 0.1542/peds.2005-2126

[5] B. Pansky and V. Ravishankar, "Biomedical gait evaluation of the immediate of orthotis treatment for flexible flat foot," Prosthetics and Orthotics International, vol.22, pp.25-34, (1997)

[6] N.J. Giannestras, "Recognition and treatment of flatfoot in infancy," Clin Orthop Relat Res, vol.70, pp.10-29, (1992)

[7] H.R. Tax, "Flexible flatfoot in children," J Am Podiatr Med Assoc, vol.67, pp.616-619, (1997)

[8] S. Sinha, H. R. Song, H. J. Kim, M. S. Park, Y. C. Yoon, and S. H. Song, "Medial arch orthosis for paeditric flatfoot," Journal of Orthopedic Surgery, vol.21, no.1, pp.37-43, (2013)

[9] D. Robert Kuhn DC, Nofa J. Shibley DC, Wiliam M. Austin DC, and Terry R. Yochum DC, "Radiographic evaluation of weight-bearing and their effect on flexible pes planus," Journal of Manipulative and Physiological Therapeutics, vol.22, no.2, pp.44-52, (1999)

[10] H. W. Perry, "Arch index as a predictor of arch height," vol.76, pp.76-85, (1999)

[11] D. Kanatli and U. Iskian, "The values of talonavicular angles in patients with pes planus," Foot Ankle surg, vol.32, no.5, pp.514-516, (1993) 
[12] M. L. Root, "Normal and abnormal functioning of the foot," Clinical Biomechanics, vol.2, pp.26-62, (1977)

[13] J. LeLievere, "Current concepts and correction in the valgus foot," Clinical Orthopedics and Related Research, vol.70, pp.43-55, (1970)

[14] S. H. Lee, H. D. Kim, and N. J. Lee, "Change of radiologic indicators during putting foot orthosis on flatfoot in children with cerebral palsy," Annals of Rehabilitation Medicine, vol.28, no.6, pp.542-548, (2004)

[15] D.G. Kulcu, K.A. Lai, and S. Sarmer, "Immediate effects of silicone insoles on gait patterns in patients with flexible flat foot," Foot Ankle Int, vol.28, no.10, pp.1053-57, (2007) DOI: 10.3113/FAI.2007.1053

[16] J. W. Kim, E. H. Lee, K. H. Ko, and S. Y. Kim, "The effect of navicular drop on the clinical measures of lower extremity alignment," The Journal of Korean Academy of Orthopedic Manual Physical Therapy, vol.16, no.1, pp.1-8, (2010)

[17] Y. S. Yoon, J. Y. Kang, S. B. Yun, J. S. Choi, J. L. Choi, K. P. Yu, and S. H. Kwak, "Effect of the foot orthosis for children scoliosis caused by inequality of resting calcaneal stance position angle", Annals of Rehabilitation Medicine, vol.34, no.1, pp.66-73, (2010)

[18] R. L. Blake, and H. Ferguson, "The inverted orthotic technique: a practical discussion of an orthotic therapy," J Br Podiatr Med, vol.48, pp.25-29, (1993)

[19] S. Roth, A. Roth, and Z. Jotanovic, "Navicular index for differentiation of flatfoot from normal foot," International orthopaedics, vol.37, pp.1107-1112, (2013) DOI: 10.1007/s00264-013-1885-6 
Effect of A Navicular Support Foot Orthosis on Resting Calcaneal Standing Position Angle and Navicular Height in Flat Foot Children

This page is empty by intention. 